\title{
RECENT ADVANCES IN MICROBIOLOGICAL METHODS
}

$\mathrm{V}$ ARIOUS aspects of recent advances in microbiological methods were discussed at a joint meeting of the Society for Applied Bacteriology and the Microbiological Group of the Society of Chemistry and Industry, which was held at the Institution of Civil Engineers, Great George Street, London, on January 14. Dr. J. G. Davis presided at the meeting.

Prof. M. Stacey opened the meeting with a paper on the "Chemistry of Gram Staining and of the Feulgen and Dische Reactions for Nuclear Material". Mention was made of the need to put the important microbiological and histological staining techniques on to a firm chemical basis. Profound chemical differences exist between the two groups of organisms: the Gram-positives, which can be stained by Gram's basic fuchsin-iodine technique; and the Gramnegatives, which do not retain the stain on being washed with alcohol. By means of bile salts Grampositives can be stripped of their surface material, leaving a Gram-negative cytoskeleton. When this is kept under reducing conditions, it is found that magnesium ribonucleate from the bile salt extract or from other sources will combine with it in such a way that the Gram-positive state can be restored. It is considered that the dye-retaining complex is a magnesium ribonucleoprotein located near the cell surface of Gram-positives. Gram-negatives, on the other hand, although possessing ribonucleic acids, have surface material composed of phospholipids, protein and polysaccharides.

The significance of ribonucleic acid in the Gramcomplex can be emphasized by the use of ribonucleases and autolytic enzymes from the cell. Cells rendered Gram-negative by the action of lytic enzymes are difficult to reconstitute unless they are first treated with the protein component of the Gram-complex, followed by addition of the magnesium ribonucleate. Mechanical disruption of cell surfaces completely destroys the possibility of reconstituting the Gramcomplex.

Deoxyribonucleic acid plays no part in the Gramcomplex ; but its deoxy sugar component is implicated in both the Dische diphenylamine and Feulgen reactions for nuclear material. The Dische reaction is explained by the finding that 2-deoxypentoses under the strongly acidic conditions of the test are transformed into $\omega$-hydroxy lævulic aldehyde that combines with diphenylamine to give a blue dye which, in the Spekker, has a characteristic band with a single peak.

The Feulgen test can be explained as a result of extensive investigations on the properties of 2deoxy sugars. Under mildly acidic conditions the 2-deoxyribofuranose component of the nuclear material readily loses some of the groups attached at the reducing carbon atom, and the sugar ring opens to give an aldehydo-sugar. This gives an intense insoluble dye with the Schiff's reagent used in the Feulgen test. Further implications of the known behaviour of deoxy sugars were mentioned. In the discussion which followed, Prof. Stacey was unable to explain some of the observations of various investigators during Gram-staining, and he mentioned that magnesium is not completely specific in the Gram-replating technique.
The next speaker was Dr. E. Windle Taylor, who described the "Use of the Membrane Filter in the Bacteriological Examination of Water". He said that collodion membranes not only retain all microorganisms from a large volume of liquid, but can also be placed in contact with a substrate which conveys nutrients through the pores to the deposited bacteria so that they can be cultivated in situ. The substrate takes the form of an absorbent pad which can be soaked with concentrated nutrient. The use of the apparatus is quite simple, but sterilization of the membranes requires great care. After much trial it has been found possible, without harming the membranes, to autoclave them for ten minutes at $10 \mathrm{lb}$. sq. in. pressure if they are stacked alternately with absorbent pads and the pile wrapped in kraft paper.

The main lines of investigation were to ascertain whether the apparatus and technique could be applied to media and methods commonly employed in Britain for the bacteriological examination of water, and this has been established in a satisfactory manner. The amount of liquid that can be filtered in a given time through each membrane depends upon the amount of suspended matter, and varies from two litres in ten minutes for tap water to a quarter of a litre in one hour for sewage effluent. Doublestrength nutrient broth is used for colony counts, and figures obtained closely follow the corresponding agar counts, while in certain circumstances, such as pure chalk waters, larger volumes can be examined. Colonies first become visible after eight hours incubation and reach a maximum after eighteen hours. MacConkey broth can be used for the coliform examination, if made up in triple strength but with bromcresol-purple indicator of twelve times strength. The lactose-fermenting colonies are clearly distinguishable as pale yellow colonies on a blue background. Colonies are first seen after eight hours incubation, and a maximum number is reached after twelve hours incubation. Preliminary resuscitation of bacteria with nutrient broth and the achievement of direct Bact. coli counts are matters for further study.

Pathogenic bacteria have been successfully isolated by membrane filtration by cultivating the membrane in selenite broth, thus saving a large volume of medium; no success has yet been achieved in producing typical colonies of enteric organisms on a membrane and pad impregnated with a liquid form of Wilson-Blair medium.

The chief advantages of the technique are concentration of organisms, removal of inhibitory substances in the water itself, saving of glassware and media and, in the case of the coliform test, the avoidance of false presumptive results due to spore-forming anaerobic lactose-fermenters. There appears to be a field of exploration along three main lines to assess the applicability of filter membranes in the bacteriological examination of water : for examining a series of samples, as in a river or a lake survey; where a rapid result is required; and for isolating pathogenic organisms from sewage effluents and polluted waters.

In the lively discussion which followed, various speakers mentioned their own experiences with the membrane filters and the commercial availability 
of various types, and the general feeling was reached that the filters would prove very valuable.

In the next paper, Dr. L. A. Allen described the "Fnumeration of Bacterium coli and of Streptococcus focalis". Growth of Bact. coli on bile salts-lactose agar at $44^{\circ} \mathrm{C}$. has been found to be conditioned by a number of factors. The temperature of $44^{\circ} \mathrm{C}$. is critical for this organism, and an accurately controlled water-bath is essential. The inclusion of phosphate in the medium reduced the count of all strains tested, and some strains were virtually unable to grow under these conditions. When the bacteria are suspended in water, the proportion of the total population able to grow on the bile salts medium at $44^{\circ} \mathrm{C}$. tends to decrease as the period of suspension is prolonged. This result of attenuation can be largely overcome by dividing the medium into two parts, each of double strength-lactose broth; and a solution containing the bile salts, indicator and agar -and subjecting the inoculum to a short period of incubation in the lactose broth at $37^{\circ} \mathrm{C}$. before adding the second part of the medium and incubating at $44^{\circ} \mathrm{C}$. 'Resuscitation' for one hour in this way results in a count on bile salts - lactose agar at $44^{\circ} \mathrm{C}$. which approximates to the count on Yeastrel-glucose agar at $37^{\circ} \mathrm{C}$. The film of agar is formed in a rotating bottle which can afterwards be directly immersed in a water-bath. The temperature cannot be controlled with sufficient accuracy if Petri dishes are used.

Evidence has accumulated in recent years that Str. focalis may be a useful indicator of fæcal contamination when used in conjunction with conventional tests for coliform bacteria. As a result of a similar study of the growth of this organism on 'Yeastrel' - glucose azide agar, a technique has been formulated for obtaining a colony count on this medium at $45^{\circ} \mathrm{C}$. Str. foecalis was found to be so sensitive to neutral red that it was necessary to leave this indicator out of the medium altogether. Phosphate exerts no depressing action on the count, provided it is not included with the other constituents of the medium before autoclaving. As with Bact. coli, 'resuscitation' largely overcomes the inability of attenuated cells to form colonies on this medium.

The principle of resuscitation has been applied to the determination of the most probable number of both organisms for use with samples in which the numbers of bacteria are too small to permit of an accurate colony-count. The resuscitation method has been found to give significantly higher results than existing methods for both organisms, it requires less time for completion, and it is virtually specific for the organism concerned.

Following the comments on this paper, Dr. S. T. Cowan dealt with "Microchemical Methods for the Characterization of Bacteria". He said that the reproducibility of a biochemical test depends on the ability of the organism to produce appropriate enzymes and also on the medium, temperature and duration of incubation, volatilization or utilization of the end-product, and the sensitivity of the test reagents. Bacteria are essentially complex enzyme systems and are most easily studied by letting nongrowing cell suspensions act on defined substrates. This principle can be applied to the biochemical tests used in bacteriological laboratories, one enzyme system being tested at a time. Each test depends on the action of a very heavy suspension of the organism : the heavier the suspension, the quicker the reaction; and a minimum density equivalent to $10^{9}$ Bacterium coli $/ \mathrm{ml}$. is essential.
Fermentations are carried out by mixing sugar solution, bromthymol blue and bacterial suspension and incubating at $37^{\circ} \mathrm{C}$. The method is sensitive and shows acid production by organisms which, in growing cultures, appear to lack fermentative ability. Other tests for glucose breakdown can be adapted to the micro-methods using suspensions. A micromethod for the methyl red test (ability to produce a $p \mathrm{H}$ of 5 or less from glucose) is being developed. Acetoin (acetylmethylcarbinol) can be detected readily; but, as the enzyme is adaptive, the suspension should be made from growth on a glucose medium. Hydrogen sulphide production can only be observed when there is an adequate source of sulphur available; cysteine hydrochloride is suitable for all the bacteria examined, but the test using cysteine and lead acetate paper is so sensitive that it has little differential value.

Nitrate reduction, the production of indole, and urease are other tests that can be carried out quickly with suspensions and the appropriate substrates. All the micro-methods so far developed are simple to set up and are quick in action; the specificity of the tests depends on the test reagents.

By testing each enzyme system separately, it should be possible to characterize a bacterium by its enzymic formula, much as a serologist characterizes it by an antigenic formula. The micromethods should be readily adaptable to studies on the availability of different amino-acids.

Numerous speakers commented on the paper, and the useful suggestion was made by $\mathrm{Mr}$. H. B. Hawley that the chromatographic purity of the sugars used by Dr. Cowan should be checked.

Finally, Mr. J. Tramer spoke on "Use of Bacterial Phosphatases in Differentiating Certain MicroOrganisms". Bray and King investigated, in 1943, the production of phosphatase by growing organisms as a possible aid for their differentiation. They assessed the phosphatase activity of a wide.variety of species by incorporating phenolphthalein phosphate in an egg medium. The amount of phenolphthalein liberated from the substrate by phosphatase served as an indication of the amount of enzyme produced. They found their relative simple technique of value in differentiating between members of certain genera, but thought the slow rate of hydrolysis of their substrate to be a disadvantage.

Barber and Kuper examined, in 1951, the possibility of distinguishing between pathogenic and non-pathogenic staphylococci by their respective phosphatase reactions. They used phenolphthalein diphosphoric acid which, when suitably incorporated into an agar medium, allowed good differentiation between phosphatase-negative colonies, which were found to be coagulase-negative and therefore nonpathogenic, and phosphatase-positive colonies which proved to be coagulase-positive and only rarely coagulase-negative.

Yet another substrate, disodium $p$-nitrophenylphosphate, has been shown to be useful in the estimation of phosphatase activity. It is colourless in solution but produces, on hydrolysis by phosphatase, free $p$-nitrophenol, which is yellow in neutral and alkaline solution and serves as a measure of enzyme activity. Although not heat-stable, solutions can be Seitz-filtered and incorporated into solid and liquid media. On solid media, colonies of phosphataseproducing organisms will show a yellow colour after a suitable incubation time, and liquid media will turn yellow if such organisms are present. Plates 
containing media into which disodium $p$-nitrophenylphosphate has been incorporated can be used for streaking, and it has been found practicable to flood inoculated and incubated normal milk agar plates with a small amount of a disodium $p$-nitrophenylphosphate solution. In the latter case, surface colonies of strong phosphatase-producing organisms will turn yellow in a few minutes.

The estimation can be made quantitative by incubating a measured amount of bacterial suspension with a suitably buffered or unbuffered solution of disodium $p$-nitrophenylphosphate for a certain time (half an hour to several hours) and estimating the colour developed in a comparator using permanent colour standards, or photo-electrically. Using such a procedure, a good relationship was found between phosphatase activity and gelatine liquefaction time of Voges-Proskauer positive, methyl red negative Coli-aerogenes organism. Bact. cloacae appears to be in general a non-, or only weak, phosphatase-producer, while Bact. aerogenes and also the $44^{\circ} \mathrm{C}$. positive type (Irregular VI) are strong phosphatase-producers.

In addition to the degree of phosphatase activity and its correlation to another biochemical reaction, there are certain characteristics of the bacterial phosphatase produced which may be of value in differentiating micro-organisms. Pett and Wynne have shown that the $p \mathrm{H}$ optimum may be of value in this respect, and it is suggested that the heat stability in certain media and the activity of a cell-free filtrate may also be of use.

\section{CHEMISTRY AND INTER-RELATIONSHIPS OF ENDOCRINE SYSTEMS AND THEIR PSYCHOLOGICAL EFFECTS}

A evening discussion meeting on this theme was $A$ held on February 26 by the Society for Visiting Scientists at 5 Old Burlington Street, London, W.1, Dr. A. S. Parkes (National Institute for Medical Research, Mill Hill) being in the chair.

Prof. Jean Roche (Collège de France, Paris) spoke first, his subject being the biochemical aspects of hormone actions. One of the major concepts influencing biochemical thought and research in the present century is that of bio-catalysts - substances of great importance profoundly influencing the rate of chemical reactions in the body, and effective in small or minute amounts. Within this general group are substances which were at first thought of as being of a very different nature--vitamins, hormones and enzymes. The demonstration that many, perhaps all, vitamins act by virtue of their intimate association with the enzymes concerned with fundamental metabolic processes in the body is of immense significance.

In the case of the hormones, early work was concerned mainly with their physiological, rather than with their biochemical, actions; the biochemist could approach their study only indirectly by studying their effects on tissue enzymes, and after this action had been shown to be specific, both for the hormone and the enzyme involved. For example, the administration of pestrogens to the castrate female animal produces a marked rise in the alkaline phosphatase content of the uterus, but not of other tissues. Progesterone alone has almost no effect but, given with oestrogens, prevents this rise in enzyme content. Arginase is an enzyme of great importance in the mammals, because of its part in the Krebs cycle for the conversion of ammonia to urea - the main nitrogenous excretion product in this group. More recently, androgens have been shown to increase the concentration of this enzyme in the kidney; the concentration in other organs and of other enzymes in the kidney is not affected. The response is not produced by other sterol hormones without androgenic activity. Similarly, Folley and Greenbaum have demonstrated a marked (hundred-fold) increase in the concentration of this enzyme in the mammary gland following parturition as lactation begins under hormonal influence. These and other observations suggest that tissues can respond by specific enzyme changes in response to specific hormones, and that through such studies the biochemist can make valuable contributions to the least well-known aspect of endocrine function-the mechanism of their peripheral action.

Prof. F. G. Young (Cambridge) suggested that hormones might be defined as substances liberated into the blood stream to produce changes in tissues at a distance. As Prof. Roche had already emphasized, their action is most probably exerted by their effect on the chemical processes of the receptor cells. As a parallel to thoir marked actions in chemically small amounts, the early workers in this field had difficulty in gaining general acceptance for their concepts as there was reluctance to admit that such physically insignificant organs as the pituitary and adrenal glands and the apparently unimportant islet cells of the pancreas could be of supreme importance to the maintenance of life. Full acceptance was delayed until it was shown that the disastrous results of experimental removal of these 'inner secreting' or endocrine glands could be reversed by injections of potent extracts from them. Even the central nervous system, which at the time when the early observations were made, such as that of Addison in 1855, was thought to be the dominant controlling system, has been shown to depend largely on the maintenance of the correct balance of endocrine activity for its proper functioning. The level of sugar in the circulating blood is largely under hormonal control : a doubling leads to diabetes and severe metabolic upset; a halving to coma, convulsions and death.

Chemically, the hormones are a very heterogeneous group ; even in a single gland there is often a multiple secretion rather than a single hormone. Very recently it has been shown that the thyroid, formerly thought to secrete only one effective chemical substance-the iodo-amino-acid thyroxine-probably secretes also the closely related and more active 3'-3-5-tri-iodothyronine.

In conclusion, Prof. Young pointed out that many endocrine glands are derived embryologically from 'barrier' tissues-the ectoderm separating the animal from the external environment, and the endoderm of the alimentary canal coming between the body as a whole and the gut lumen. He postulated that one important action of hormones in the tissues might be concerned with increasing or decreasing the rate at which important metabolites cross the barrier of the cell membranes.

Prof. G. W. Harris (Institute of Psychiatry, University of London) said that the profound effects 